\title{
Automatic Modulation Classification Method for Multiple Antenna System Based on Convolutional Neural Network
}

\author{
Juan Wang ${ }^{\dagger}$, Yu Wang ${ }^{\dagger}$, Wenmei $\mathrm{Li}^{\dagger}{ }^{\dagger}$, Guan Gui ${ }^{\dagger}$, Haris Gacanin ${ }^{\dagger \dagger}$, and Fumiyuki Adachi* \\ ${ }^{\dagger}$ College of Telecommunications and Information Engineering, NJUPT, Nanjing 210000/ China \\ ${ }^{\ddagger}$ School of Geographic and Biologic Information, NJUPT, Nanjing $210023 / k$ China \\ †† Electrieal Engineerimg and Information Technology, RWTH Aachen University, Aachen 55-52062, Germany \\ * Research Organization of Electrical Communication, Tohoku University, Sendai 980 8577, Japan \\ E-mails: \{1219012920, guiguan\}@njupt.edu.cn, haris@ieee.org, adachi@ecei.tohoku.ac.jp
}

\begin{abstract}
In order to transmit communication signals of different properties, quickly, effectively, and accurately, various different modulation styles can be adopted. Accurate recognition of signal modulation is required at the receive side. Automatic modulation recognition (AMR) is a key technique to identify various styles of modulation of signals received in wireless channels. It can be used in many kinds of communication systems, including single antenna system and multiple antenna system. In this paper, we propose a convolutional neural networks (CNN) aided AMR method for multiple antenna system. Compared with the high order cumulants (HOC) and artificial neural networks (ANN) aided traditional AMR classification method, both with two specific combination strategies, such as relative majority voting method and arithmetic mean method, the proposed AMR with arithmetic mean method has the best classification performance. The experimental results obtained verify that the $\mathrm{CNN}$, one of the representative algorithms of deep learning, has a strong ability to exploit dominant features and classify the modulation styles.
\end{abstract}

Index Terms-Convolutional neural network, signal recognition, multiple antenna system, deep learning, cooperative decision.

\section{INTRODUCTION}

Automatic modulation recognition (AMR) refers to recog nize the actunal modulation style of the signal received to ensure that the signal can be demodulated correctly and the information sent can be accurntely recovered, which is the basis of various applications, especinlly in cognitive radio (CR), spectrum sensing, ete [1]-[6]. The role of the $\Lambda \mathrm{MP}$ is ef importance to communication in beth the particular military and civil fields [7] AMR need to be versatile, including being able to recognize as many modulation styles as possible, being able to function with limited knowledge of the channel or target communication system, and being able to be used in multiple styles of communication systems including single antenna system [8] and multiple antenna system [9]-[13].

Until now, many AMR method have been proposed. High-precision decision theory (DT) and easy-to-implement pattern recognition (PR) are the two basic styles of these methods [10]. Although DT-based method ean have high accuracy, it not only noed to be based on signal model matching and accurate channel statement information (CSI) [14]. but alse has higher computational complexity. More PRbased methods are adopted, no need for such complicated processing and brealking away from the limitations of prior information, generally consisting of signal preprocessing, feature exploitation and classification recognition. Among these sub-systems, artificial feature extraction covers circulatory stationary analysis [15], high-order statistics (HOS), etc, and eirculatory stationary analysic, and classification identification includes k-nearest neighbor (KNN), support vector machines (SVM) and traditional artificial neural networks (ANN) [9][13]. In the background of the additive white Gaussian noise (ANGN), HOS is a popular option in single antenna and multiple antenna systems because of its powerful ability ef suppress interference [10]. AMP research for single antenna system has been widely carried out. In recent yoars, the multiple antenna system has been widely concerned, in which multiple transmitting and receiving antennas are capable of improving transmission quality of signals as well as enhancing utilization efficiency of spectrum, accordingly saticfying the increasing data rate needs of current and future wireless systems [15].

The applications of deep learning (DL) in wireless communications are increasingly widespread [16]-[20], such as beamforming [21], non-orthogonal multiple access (NOMA) [22], network traffic prediction [23]-[27], internet of things [28]-[31], and AMC [32]-[35]. The DL-based AMR methods are mainly designed for single antenna system, but is not suitable for multiple antenna system because the receiver receives mixed signals from all transmitting antennas. Few studies have been conducted on the introduction of DL, not only restriet to convolutional neural network (CNN) [33] and long short-term memory (LSTM) [36], into AMR in multiple systems. In this thesis, we propose a convolutional neural networks (CNN) aided AMR method for multiple antenna system, where $\mathrm{CNN}$ is trained according to the signals received by all antennas. Following this part, the given decision maker collaboratively determines the modulation style according to 
the identification results of each antenna obtained by CNN. Here, two kinds of combination strategies, ${ }_{\lambda}$ the relative majority voting method and the arithmetic mean method, are adopted. Comparison result shows that the classification performance of proposed AMR method is better than that of the HOC and ANN aided AMR method in [12], [37]. Moreover, the arithmetic mean method is always better than the relative majority voting method in the two proposed combination strategy.

The rest of this paper is organized as below. In Section II, we introduce the system model and dataset generation. In Section III, we present the proposed CNN based $\Lambda M C$ method for multi antenna system. Section IV provides simulation results to evaluate the proposed method. In Section V, we conclude this paper and point out some future work.

\section{System Model And DAtaset Generation}

\section{A. System Model}

The multiple antenna system, which utilizes the air separation transmitting and receiving antenna arrays, has become the key technology of many innovative communications in recent years. Multiple antenna systems transmit multiple signal streams through different signal paths in space, known as space division multiplexing (SDM), and provide the possibility to improve link reliability. Since there are multiple paths between the transmitting antenna array and the receiving antenna array, each receiver receives a mixed signal symbol from all transmitters, so it is no longer applicable in a single antenna system.

The multiple antenna system consists of $N_{t}$ transmit antennas and $N_{r}$ receive antennas. Consider a time-invariant Rayleigh fading channel whose channel matrix $\boldsymbol{H}$ is given by $N_{r} \times N_{t}$ complex matrix. In the case of complete synchronization, in the total observed $N$ samples, the sample vector $\boldsymbol{r}_{k}=\left[r_{k}(1), r_{k}(2), \cdots, r_{k}\left(N_{r}\right)\right]^{T}, k \in[1, N]$ of the $k$-th received signal can be given by

$$
\boldsymbol{r}_{k}=\boldsymbol{H} \boldsymbol{s}_{k}+\boldsymbol{w}_{k}
$$

where $s_{k}=\left[s_{k}(1), s_{k}(2), \cdots, s_{k}\left(N_{t}\right)\right]^{T}, k \in[1, N]$ is the $k$-th transmitted signal symbol vector. $\boldsymbol{w}_{k}=$ $\left[w_{k}(1), w_{k}(2), \cdots, w_{k}\left(N_{t}\right)\right]^{T}, k \in[1, N]$ is the observed additive noise from the $k$-th signal sample. It is assumed that the transmitted symbol vectors are independent and identically distributed, and each modulation symbol has the same probability, and furthermore, the additive noise is AWGN with zero mean and one variance, i.e. $w_{k} \in N\left(0, I_{N_{r} \times N_{r}}\right)$.

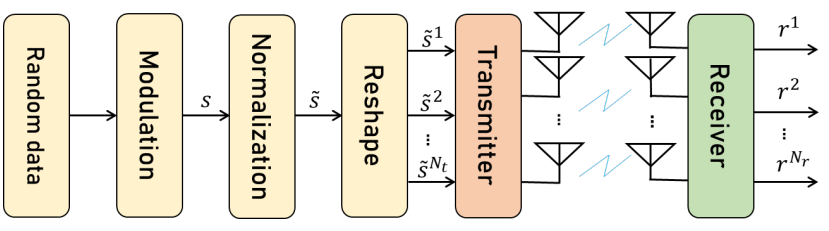

Fig. 1. Data set formation process for multiple antenna system.

\section{B. Dataset Generation}

Fig. 1 shows the specific data set formation process, where $s$ is a data sequence with dimension is $1 \times N$ formed by modulation of randomly generated data. The normalization is to speed up convergence and make the signals of different modulation styles have better differentiation. After reshaping, $\tilde{\boldsymbol{s}}^{i}=\left[\tilde{s}_{1}(i), \tilde{s}_{2}(i), \cdots, \tilde{s}_{N / N_{t}}(i)\right]^{T}, i \in\left[1, N_{t}\right]$, represents $N / N_{t}$ signals of the $i$-th transmitting antenna. Accordingly, $\boldsymbol{r}^{j}=\left[r_{1}(j), r_{2}(j), \cdots, r_{N / N_{t}}(j)\right], j \in\left[1, N_{t}\right]$ represents $N / N_{t}$ complex-value baseband signal at the $j$-th receiving antenna after passing through wireless channel. The real part and imaginary part of $\boldsymbol{r}^{j}$ will be set as a set of training sample of the $j$-th antenna.

\section{The Proposed AMC Method}

The classification decision is to find the candidate modulation style that provides the maximum likelihood value, which can be calculated by

$$
\hat{m}_{n}^{j}=\arg \max _{n \in[1,|\mathcal{M}|]} P\left(m_{n} \mid r^{j}\right), j \in\left[1, N_{r}\right],
$$

where $P^{j}=\left\{P\left(m_{n} \mid r^{j}\right)\right\}_{n=1}^{|\mathcal{M}|}$ is the received antenna $N_{r}$ probability distribution function (PDF). $\hat{m}_{n}^{j}$ and $m_{n}$ represents the predicted modulation type and the real modulation type, respectively. Here, the modulation signal types are set as $\mathcal{M}=$ $\{B P S K, Q P S K, 8 P S K, 16 Q A M\}$ [32], and the number of its internal modulation styles is expressed in $|\mathcal{M}|$.

Fig. 2 shows the structure of proposed AMC method, containing a designed $\mathrm{CNN}$ and a specific decision maker. The testing process can be divided into two stages. First, the signals $r^{j}$ received by each antenna are input into the trained CNN to extract the effective features and give the predicted PDFs $\left\{P^{j}\right\}_{j=1}^{N_{r}}$. It is important to note that the CNN here is the central training, which means to train $\mathrm{CNN}$ together according to the received signals of all the receiving antennas, rather than to train their own CNN separately with the received signals of each antenna. Then, the specific decision maker will decide whether to receive the PDFs or the sub-results of each receiving antenna according to the selected decision rule, and jointly give the final prediction style.

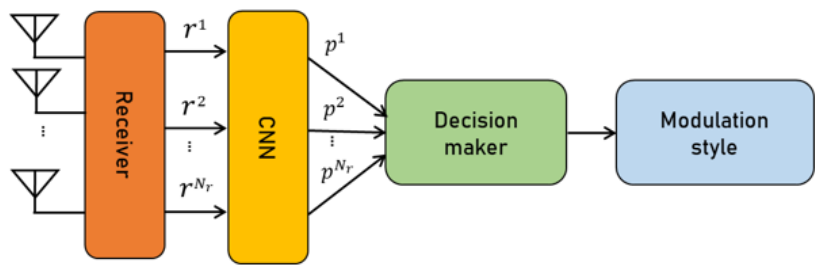

Fig. 2. Architecture of proposed AMR method.

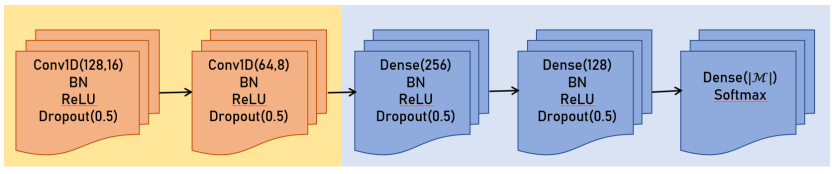

Fig. 3. The framework of CNN. 


\section{A. CNN Structure}

As shown in Fig. 3, the CNN has five layers with two convolutional (i.e. conv1D) layers and three full connected (i.e. Dense) layers. Batch normalization (BN) allows for faster convergence and shorter overall training time. To prevent overtraining the network from leading to overfitting, we set dropout $=0.5$. Based on our training dataset and CNN, the final optimization function, i.e. structural risk minimization (SRM), can be described as

$$
\begin{aligned}
& L\left(f_{C N N}, \theta ;\left\{s_{i}, l_{i}\right\}_{i=1}^{S}\right)= \\
& \min \left[-\frac{1}{S} \sum_{i=1}^{S} \log \left(f_{C N N}\left(\theta ; s_{i}\right)\right)+\lambda J\left(f_{C N N}, \theta\right)\right]
\end{aligned}
$$

where $\left\{s_{i}, l_{i}\right\}_{i=1}^{S}$ represents the combination of training dataset containing $S$ training samples and their corresponding one-hot coded-labels; $f_{C N N}$ and $\theta$ are the mapping function and parameters of the $\mathrm{CNN}$, respectively. $\min \left[-1 / S \sum_{i=1}^{S} l_{i} \log \left(f_{C N N}\left(\theta ; s_{i}\right)\right)\right]$ represents empirical risk minimization (ERM) for the classification problem. $J\left(f_{C N N}, \theta\right)$ is a function of the complexity of the model, and $\lambda \geq 0$ is a coefficient used to weigh empirical risk and the complexity of model. Adaptive moment estimation (ADAM) is selected as the optimizer [10].

\section{B. Combination Strategy}

The specific decision maker jointly determines the modulation style according to the PDFs of the receiving antenna $N_{r}$. The PDF here is the product of the signal samples to be tested obtained by designed CNN. Especially, the joint decision is because the proposed AMR method is based on two combination strategies, namely relative majority voting method and arithmetic mean method. It is obvious from relative majority voting method that the modulation style with the most predicted sub-result will be determined as the final classification result. It is important to note that if more than one category wins the highest vote, one category is randomly selected to be the final category. The arithmetic mean method is the arithmetic mean of the PDFs of the receiving antenna and the final decision result depends on the one with the highest probability of the modulation style. The detailed description of relative majority voting method and arithmetic mean method is listed in Algorithm 1.

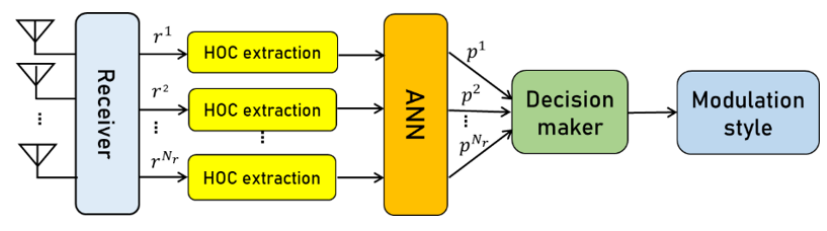

Fig. 4. Architecture of HOC and ANN aided traditional AMR method.

\section{Traditional HOC and ANN aided AMR Methods}

Different modulation styles have different cumulative values, so the classification of modulation styles can be $\overline{\text { Algorithm } 1 \text { The proposed AMR based on two combination }}$ strategy.

Require: Test sample $\left\{\boldsymbol{r}^{j}\right\}_{j=1}^{N_{r}}$ and the trained CNN;

Ensure: The predicted modulation type;

1: for $j=1: N_{r}$ :

Give the $\hat{m}^{j}$ by (2) and

$\boldsymbol{P}^{j}=\left[P\left(m_{1} \mid r_{j}\right), P\left(m_{1} \mid r_{j}\right), \cdots, P\left(m_{|\mathcal{M}|} \mid r_{j}\right)\right]$

end

2: if choosing relative majority voting method,

$\hat{M}=\frac{1}{N_{r}} \sum_{j=1}^{N_{r}} \hat{m}_{n}^{j}$;

$\hat{m}_{n}^{\text {voting }}=\arg \max _{n \in[1,|\mathcal{M}|]} \hat{M}(n)$;

end

3: if choosing arithmetic mean method,

$\hat{P}=1 / N_{r} \sum_{j=1}^{N_{r}} P_{j}$;

$\hat{m}_{n}^{\text {averaging }}=\arg \max _{n \in[1,|\mathcal{M}|]} \hat{P}(n) ;$

return The predicted modulation type $\hat{m}^{\text {Voting }}$ or $\hat{m}^{\text {Averaging }}$

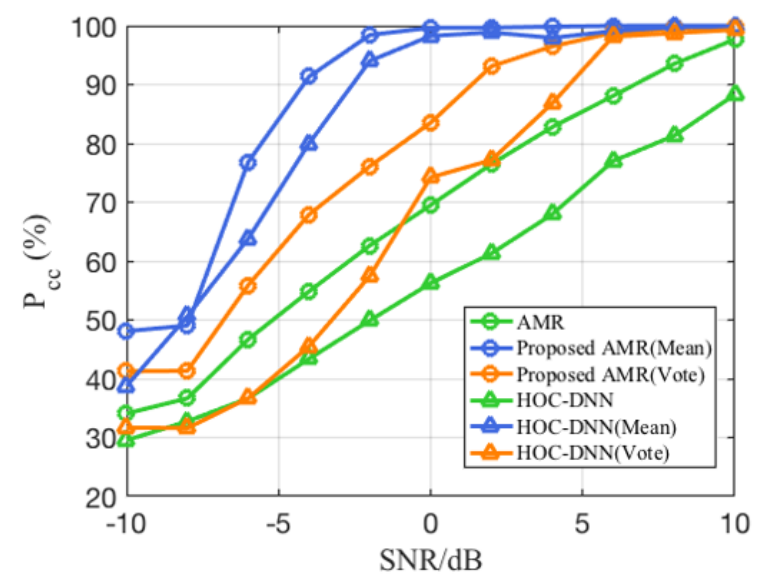

Fig. 5. The performance of classification of proposed AMR method and the HOC and ANN aided traditional AMR methods with two specific combination strategies.

realized. Swami and Sadler suggest to classify M-PAM, MPSK, and M-QAM modulation by charactering the fourthorder cumulants of complex signals [38]. Here, we use a traditional AMR based on ANN and HOC [12], [37] to make a comparison, whose architecture is shown in Fig. 4. Traditional AMR also uses central training and cooperative decision to be fair. In addition, ANN is a classifier having three fullyconnected whose parameters are consistent with the fullyconnected layer of proposed AMR.

\section{Simulation Results}

In this section, we demonstrate that the proposed AMR method for multiple antenna system has better classification performance through simulation. For each modulation style, 20,000 samples are prepared per signal-to-noise ratio (SNR) for training CNN network, the training set and validation set reasonably divided according to $7: 3$, and 10,000 samples are prepared per SNR for testing to obtain the correct classification probability $P_{c c}$ at $s n r \mathrm{~dB}$.

$$
P_{c c}=\frac{S_{\text {correct }}^{\text {snr }}}{S_{\text {test }}} \times 100 \%
$$




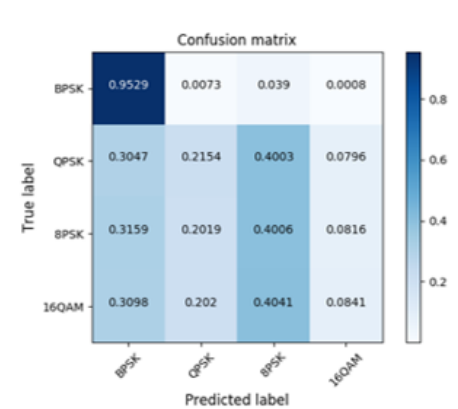

$s n r=-8 \mathrm{~dB}$

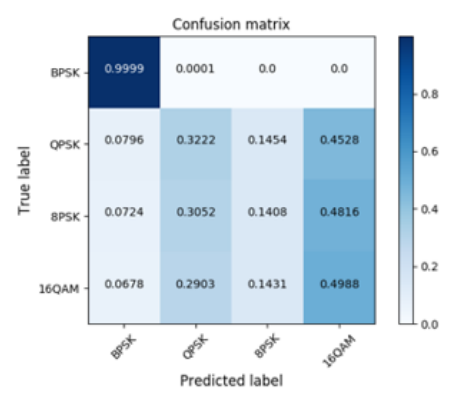

$s n r=-8 \mathrm{~dB}$

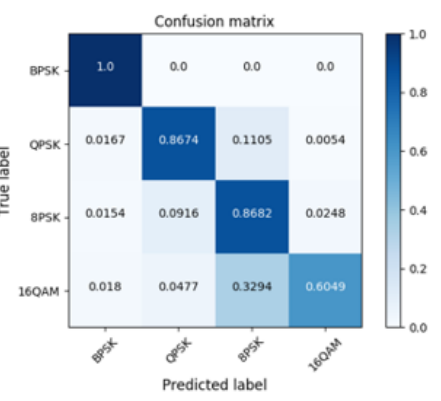

$s n r=0 \mathrm{~dB}$

(a) voting method

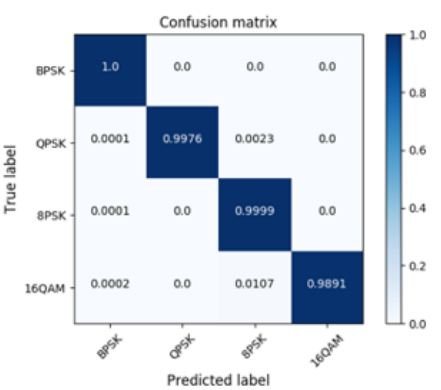

$s n r=0 \mathrm{~dB}$

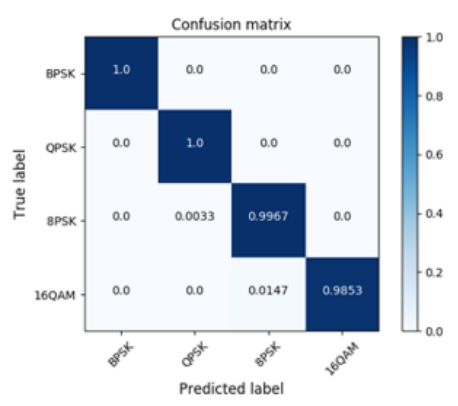

$s n r=8 \mathrm{~dB}$

(b) averaging method

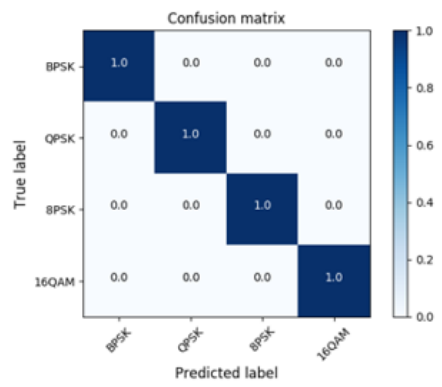

$s n r=8 \mathrm{~dB}$

Fig. 6. Confusion matrices of CNN using (a) relative majority voting method and (b) arithmetic mean method when $s n r \in\{-8,0,8\} \mathrm{dB}$.
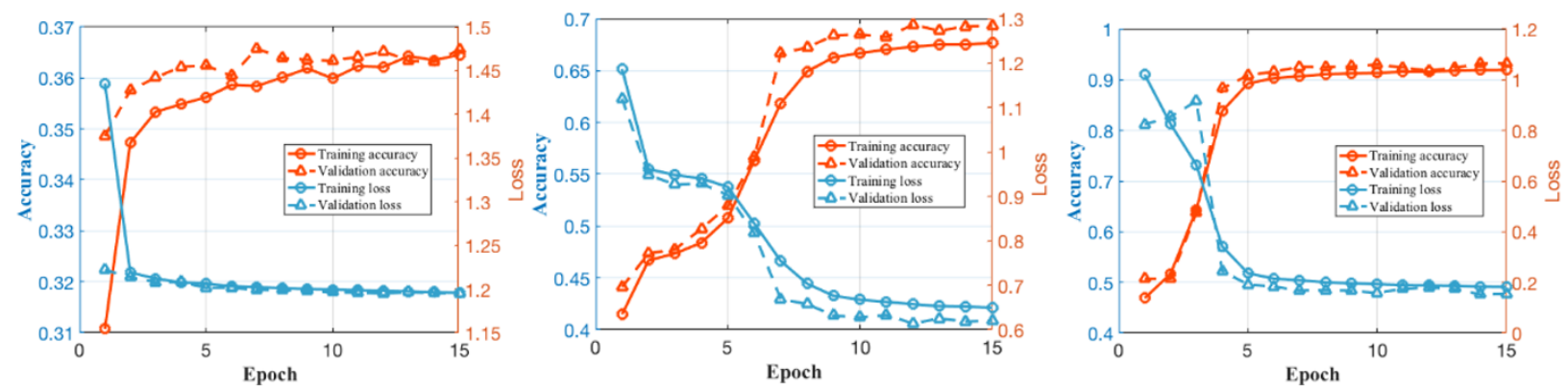

Fig. 7. The loss and accuracy curves of training validation.

where $S_{\text {test }}$ and $S_{\text {correct }}^{\text {snr }}$ represent the number of samples of each modulation style and the correct number of classification of all modulation styles at snr $\mathrm{dB}$, respectively. The performance of classification of proposed AMR method and the HOC and ANN aided traditional AMR methods with two specific combination strategies under different SNR are showed in Fig. 5. Here, we design $N_{r}=4$ and $N_{t}=1$. AMR in 5 represents the average probability of correct classification of four received antennas. It is obvious that the arithmetic mean method is always better than the relative majority voting method in both proposed AMR method and the HOC and ANN aided traditional AMR methods. In condition, through the comparison of the two AMR methods, the classification performance of proposed AMR has been greatly improved in two kinds of combination strategies.
Furthermore, we provide six confusion matrices of proposed AMR method using relative majority voting method and arithmetic mean method when snr $\in\{-8,0,8\} \mathrm{dB}$ in Fig. 6 respectively, which helps to analyze the performance of our algorithm in detail. We can see that proposed AMR using arithmetic mean method can almost accurately identity BPSK modulation even when $s n r=-8 \mathrm{~dB}$, and it can completely identity four modulation styles when $s n r=8 \mathrm{~dB}$, which is also better than the relative majority voting method. The accuracy and loss curves of training and validation are also provided in Fig. 7 to prove that we do not overtrain the network just to get the desired results and lead to overfitting. 


\section{CONCLUSION}

In this paper, we have proposed CNN aided AMR method for multiple antenna system. Our approach adopted two combination strategies: relative majority voting method and arithmetic mean method. Experimental results show that the arithmetic mean method has better classification performance than the relative majority voting method. Comparing with the HOC and ANN aided traditional AMR methods with the same two combination strategies under the same fully-connected layer parameters, the proposed AMR method with the arithmetic mean method has best classification performance, which shows that $\mathrm{CNN}$ has the advantage of dominant feature exploitation and high recognition accuracy, compared with the traditional artificial feature designing methods. In future work, we plan to propose more advanced method by using transfer learning and federated learning algorithms.

\section{REFERENCES}

[1] F. Meng, P. Chen, L. Wu and X. Wang, "Automatic modulation classification: a deep learning enabled approach," IEEE Trans. Veh. Technol., vol. 67, no. 11, pp. 10760-10772, Nov. 2018.

[2] Y. Wang, M. Liu, J. Yang and G. Gui, "Data-driven deep learning for automatic modulation recognition in cognitive radios," IEEE Trans. Veh. Technol., vol. 68, no. 4, pp. 4074-4077, 2019.

[3] M. Liu, T. Song, H. Hu, J. Yang and G. Gui, "Deep learning-inspired message passing algorithm for efficient resource allocation in cognitive radio networks," IEEE Trans. Veh. Technol., vol. 69, no. 1, pp. 641-653, Jan. 2019

[4] B. Ramkumar, "Automatic modulation classification for cognitive radios using cyclic feature detection," IEEE Circuits Syst. Mag., vol. 9, no. 2, pp. 27-45, 2009.

[5] N. Kato, B. Mao, F. Tang, Y. Kawamoto, and J. Liu, "Ten challenges in advancing machine learning technologies towards 6G," IEEE Wireless Commun. Mag., in press, doi: 10.1109/MNET.001.1900476

[6] F. Tang, Y. Kawamoto, N. Kato, and J. Liu, "Future intelligent and secure vehicular network towards 6G: Machine-learning approaches," Proc. IEEE, vol. 108, no. 2, pp. 292-307, Feb. 2020.

[7] G. Gui, M. Liu, F. Tang, N. Kato, and F. Adachi, "6G: Opening new horizons for integration of comfort, security and intelligence," IEEE Wireless Commun. Mag., in press, doi: 10.1109/MWC.001.1900516.

[8] T. O'Shea and J. Hoydis, "An introduction to deep learning for the physical layer," IEEE Trans. Cogn. Commun. Netw., vol. 3, no. 4, pp. 563-575, Apr. 2017.

[9] K. Hassan, I. Dayoub, W. Hamouda, C. Nzeza and M. Berbineau, "Blind digital modulation identi?cation for spatially-correlated MIMO systems," IEEE Trans. Wirel. Commun., vol. 11, no. 2, pp. 683-693, 2011.

[10] Y. Wang, J. Wang, W. Zhang, J. Yang, and G. Gui, "Deep learning-based cooperative automatic modulation classification method for MIMO systems," IEEE Trans. Veh. Technol., in press, doi: 10.1109/TVT.2020.2976942

[11] D. Das, P. K. Bora and R. Bhattacharjee, "Blind modulation recognition of the lower order PSK signals under the MIMO keyhole channel,", IEEE Commun. Lett., vol. 22, no. 9, pp. 1834-1837, Sept. 2018.

[12] K. Hassan, I. Dayoub, W. Hamouda, C. Nzeza and M. Berbineau, "Blind digital modulation identification for spatially-correlated MIMO systems," IEEE Trans. Wirel. Commun., vol. 11, no. 2, pp. 683-693, Feb. 2011

[13] S. Kharbech, I. Dayoub, M. Zwingelstein-Colin, and K. Hassan, "Blind digital modulation identification for time-selective MIMO channels," IEEE Wirel. Commun. Lett., vol. 3, no. 4, pp. 373-376, Apr. 2014.

[14] F. Hameed, O. A. Dobre, and D. C. Popescu, "On the likelihood-based approach to modulation classification," IEEE Trans. Wirel. Commun., vol. 8, no. 12, pp. 5884-5892, Dec. 2009.

[15] M. S. Muhlhaus, M, Oner, O. A. Dobre, H. U. Jkel, and F. K. Jondral, "Automatic modulation classification for mimo systems using fourthorder cumulants," in IEEE Vehicular Technology Conference (VTC Fall), Quebec City, QC, Canada, 3-6 Sept. 2012, pp. 1-6.

[16] R. W. Heath, "Going toward 6G," IEEE Signal Process. Mag., vol. 36, no. 3, pp. 3-4, 2019 .
[17] K. B. Letaief, W. Chen, Y. Shi, J. Zhang, and Y. A. Zhang, "The roadmap to 6G-AI empowered wireless networks," IEEE Commun. Mag., vol. 57, no. 8, pp. 84-90, 2019.

[18] Z. Qin, H. Ye, G. Y. Li, and B. Juang, "Deep learning in physical layer communications," IEEE Wireless Commun. Mag.. vol. 26, no. 2, pp. 93-99, 2019.

[19] H. He, S. Jin, C.-K Wen, F. Gao, G. Y. Li, and Z. Xu, "Modeldriven deep learning for physical layer communications," IEEE Wireless Commun. Mag.. vol. 26, no. 5, pp. 77-83, 2019.

[20] J. Sun, W. Shi, Z. Han, J. Yang, and G. Gui, "Behavioral modeling and linearization of wideband RF power amplifiers using BiLSTM networks for 5G wireless systems," IEEE Trans. Veh. Technol., vol. 68, no. 11, pp. 10348-10356, 2019.

[21] H. Huang, Y. Peng, J. Yang, W. Xia and G. Gui, "Fast beamforming design via deep learning," IEEE Trans. Veh. Technol., vol. 69, no. 1, pp. 1065-1069, Jan. 2020.

[22] N. Ye, X-M. Li, H. Yu, L. Zhao, W. Liu, X. Hou, "DeepNOMA: A unified framework for NOMA using deep multi-task learning," IEEE Trans. Wireless Commun., doi: 10.1109/TWC.2019.2963185

[23] G. Gui, F. Liu, J. Sun, J. Yang, Z. Zhou, and D. Zhao, "Flight delay prediction based on aviation big data and machine learning," IEEE Trans. Veh. Technol., vol. 69, no. 1, pp. 1065-1069, 2020.

[24] X. Sun, S. Ma, Y. Li, D. Wang, Z. Li, N. Wang and G. Gui, "Enhanced echo-state restricted boltzmann machines for network traffic prediction," IEEE Internet Things J., vol. 7, no. 2, pp. 1287-1297, 2020.

[25] B. Mao, F. Tang, Z. Md. Fadlullah, and N. Kato, "A novel nonsupervised deep learning based network traffic control method for software defined wireless networks," IEEE Wireless Commun. Mag., vol. 25 , no. 4 , pp. 74-81, 2018.

[26] F. Tang, B. Mao, Z. Md. Fadlullah, N. Kato, O. Akashi, and T.Inoue, and Kimihiro Mizutani, , "On removing routing protocol from future wireless networks: A real-time deep learning approach for intelligent traffic control," IEEE Wireless Commun. Mag., Vol. 25, no. 1, pp. 154160, Feb. 2018.

[27] N. Kato, Z. Md. Fadlullah, B. Mao, F. Tang, O. Akashi, T. Inooue, and K. Mizutani, "The deep learning vision for heterogeneous network traffic control: Proposal, challenges, and future perspective," IEEE Wireless Commun. Mag., vol. 24, no. 3, pp. 146-153, 2017.

[28] M. Liu, T. Song, G. Gui, J. Hu, and H. Sari, "Deep cognitive perspective: Resource allocation for NOMA based heterogeneous IoT with imperfect SIC," IEEE Internet Things J., vol. 6, no. 2, pp. 2885-2894, Apr. 2019.

[29] F. Tang, B. Mao, Z. Md. Fadlullah, and N. Kato, "On a novel deep learning based intelligent partially overlapping channel assignmentin SDN-IoT," IEEE Commun. Mag., vol. 56, no. 9, pp. 80-86, Sept. 2018.

[30] H. Liang, X. Zhang, J. Zhang, Q. Li, S. Zhou, and L. Zhao, "A novel adaptive resource allocation model based on SMDP and reinforcement learning algorithm in vehicular cloud system," IEEE Trans. Veh. Technol., vol. 68, No. 10, pp. 10018-10029, Oct. 2019.

[31] B. Zheng, P. He, L. Zhao, and H-W. Li, "A hybrid machine learning model for range estimation of electrical vehicles," IEEE GLOBECOM, Washington DC, USA, 4-8 Dec. 2016, pp. 1-6.

[32] Y. Wang, J. Gui, Y. Yin, J. Wang, J. Sun, G. Gui, H. Gacanin, H. Sari, and F. Adachi, "Automatic modulation classification for MIMO systems via deep learning and zero-forcing equalization," IEEE Trans. Veh. Technol., in press, 2020.

[33] Y. Wang, J. Yang, M. Liu, and G. Gui, "LightAMC: Lightweight automatic modulation classification using deep learning and compressive sensing," IEEE Trans. Veh. Technol., in press, doi: 10.1109/TVT.2020.2971001

[34] Y. Tu and Y. Lin, "Deep neural network compression technique towards efficient digital signal modulation recognition in edge device," IEEE Access, vol. 7, no. 1, pp. 58113-58119, 2019.

[35] Y. Tu, Y. Lin, J. Wang, and J.-U. Kim, "Semi-supervised learning with generative adversarial networks on digital signal modulation classification," Comput. Mater. Continua, vol. 55, no. 2, pp. 243-254, May. 2018.

[36] S. Hu, Y. Pei, P. Lian and Y. Liang, "Deep neural network for robust modulation classification under uncertain noise conditions," IEEE Trans. Veh. Technol., vol. 69, no. 1, pp. 564-577, Jan. 2020.

[37] M. Abdelbar, B. Tranter, and T. Bose, "Cooperative modulation classification of multiple signals in cognitive radio networks," in IEEE ICC, Sydney, Australia, June 10-14, 2014, pp. 1483-1488.

[38] A. Swami and B. M. Sadler, "Hierarchical digital modulation classification using cumulants," IEEE Trans. Commun., vol. 48, no. 3 , pp. 416-429, 2000. 